\title{
Italie : une promesse non tenue !
}

Italy: a broken promise!

Italia : una promesa no cumplida!

\section{Gianni Colombo}

Traducteur : Manuela Retailleau

\section{(2) OpenEdition}

12 Journals

Édition électronique

URL : http://journals.openedition.org/ries/2795

DOI : 10.4000/ries.2795

ISSN : 2261-4265

Éditeur

Centre international d'études pédagogiques

Édition imprimée

Date de publication : 1 mars 1999

Pagination : 65-69

ISBN : 9771254459005

ISSN : $1254-4590$

\section{Référence électronique}

Gianni Colombo, «Italie : une promesse non tenue! », Revue internationale d'éducation de Sèvres [En ligne], 21 | mars 1999, mis en ligne le 16 avril 2013, consulté le 08 janvier 2020. URL : http:// journals.openedition.org/ries/2795; DOI : 10.4000/ries.2795

Ce document a été généré automatiquement le 8 janvier 2020

(c) Tous droits réservés 


\section{Italie : une promesse non tenue!}

Italy: a broken promise!

Italia : una promesa no cumplida!

\section{Gianni Colombo}

Traduction : Manuela Retailleau

1 Dans la quasi totalité des pays européens, les problèmes de l'accès à l'enseignement et de son exercice sont posés et résolus en rapport avec les compétences requises des enseignants.

2 Le texte du rapport signé par les participants de la $\mathrm{XV}^{e}$ conférence des ministres de l'Éducation ${ }^{1}$, tenue à Helsinki en 1987, précise que «La formation initiale des enseignants doit être ancrée sur une culture générale vaste et solide.

3 Elle doit développer des capacités intellectuelles qui puissent permettre aux enseignants de relever les nouveaux défis de leur future fonction et de privilégier les savoirs essentiels dans la masse des informations disponibles.» La formation initiale doit mettre l'accent sur l'acquisition de compétences humaines et sociales, la pratique pédagogique, la connaissance du système scolaire et de son fonctionnement, la maitrise des disciplines et leur préparation didactique - c'est-à-dire la compréhension de la méthode par laquelle leur contenu peut être trié, organisé et transmis - sans omettre une réflexion sur les valeurs et leur diffusion à la jeunesse des sociétés pluralistes européennes.

4 Il faut cependant reconnaître qu'en Europe, le corps enseignant est encore loin de bénéficier d'une formation répondant à la diversité des objectifs si soigneusement définis par les responsables nationaux de l'instruction.

5 Mais quelle position l'Italie occupe-t-elle vis-à-vis de ses voisins européens? Si la formation initiale peut être considérée comme un point acquis en Europe, l'est-elle en Italie?

6 La réponse est simple : le classement de l'Italie dans le domaine de la formation est encore moins bon que celui de son économie; la formation semble être à l'image de l'Italie elle-même, aussi prometteuse que défaillante; les déclarations de principe épuisées, il ne reste que le vide ; on compte sur la disponibilité et la bonne volonté des 
intéressés qui, dans l'inconfort et les difficultés, continuent leur traversée en mer hostile.

7 Les lois, les contrats et les engagements à ce sujet sont innombrables ; cependant, vingt ans après le décret présidentiel 417/74, ratifiant une formation universitaire complète pour tous les enseignants, la formation initiale demeure une promesse non tenue.

8 Alors que la plupart des pays européens prévoient de modifier de façon significative leurs systèmes de formation pour y inclure trois, voire quatre années d'études au niveau universitaire ou para-universitaire, l'Italie est encore le seul pays d'Europe où quatre ans de cycle secondaire supérieur suffisent pour enseigner dans le primaire et où seulement trois années de ce même cycle permettent l'exercice en maternelle. En outre, être enseignant à 17-18 ans en Italie signifie rester dans l'attente d'une affectation durant un temps illimité, accumuler minutieusement les moindres services, mêmes les plus fatigants et les plus coûteux pour atteindre le barème minimal d'accès à un emploi épisodique et précaire.

9 L'espoir et la surprise de la rentrée 1998 c'est l'apparition d'une formation initiale dans quelques universités italiennes qui créent, pour la première fois, des départements de sciences de l'éducation et des facultés des sciences de l'enseignement primaire et secondaire. Leurs cursus, à compter de l'année académique quatre-vingt-dix-huit/ quatre-vingt-dix-neuf, prévoient en alternance sur quatre ans des cours théoriques de socio-psycho-pédagogie et des stages de pratique guidée.

10 C'est un événement majeur compte tenu des années d'attente au cours desquelles érudits et chercheurs ont formulé nombre de propositions demeurées sans réponse auprès des différents gouvernements qui se sont succédé depuis la mise en vigueur du DPR 417/74.

11 Ces créations ouvrent également des perspectives intéressantes en faveur des écoles qui travaillent à accroître leur autonomie financière et didactique : leur lien avec l'université chargée de proposer aux futurs enseignants des stages de pratique guidée favorisera aussi la formation continue des enseignants tuteurs. Ces derniers, à leur tour, pourront être les initiateurs de projets adaptés à leur rôle, favorisant ainsi l'innovation et la mise en œuvre de processus efficaces au sein de l'école, de même qu'entre les divers ordres et degrés du système éducatif.

\section{La formation continue en Italie : une farce de plus ?}

On ne peut qu'être surpris de constater que la loi du 11 août $1991^{2}$ constitue encore la référence juridique dont le ministère de l'Instruction publique se prévaut pour élaborer un plan national de formation continue qui disperse ce qui a été laborieusement assemblé ces dernières années.

13 En effet, l'article 2 des dites dispositions est inclus dans un ensemble dont il diffère par nature comme le révèle l'objet même de la loi : on imagine difficilement quel rapport logique associe les décharges syndicales rémunérées et le plan national de formation continue. Il s'agit en fait d'un énième triste tour de l'appareil bureaucratique : feindre de promouvoir et de soutenir l'innovation pour renforcer son propre pouvoir et contrôler les destinataires de la formation. C'est là le véritable objectif dissimulé sous le couvert des accords syndicaux. L'alinéa 1 de l'article 2 parvient même à faire pâlir les 
plus fins connaisseurs des complexes mécanismes administratifs de l'État. On y lit textuellement que :

«Dans le cadre du plan national de formation continue et dans les limites des crédits budgétaires annuels, des fonds peuvent être directement alloués à des institutions scolaires et éducatives de tous ordres et degrés, que celles-ci soient ou non dotées de personnalités juridiques, pour la réalisation d'activités de formation continue destinées au personnel de ces mêmes institutions ou d'autres institutions scolaires. »

14 Ce qui compte, semble-t-il, c est de repartir les ressources et cela même au profit d'institutions non habilitées à effectuer des opérations à caractère administratif ou comptable pourvu que cette répartition s'opère, de façon faussement équitable, selon l'importance de leur participation respective.

Par la suite, et concernant les dispositions de nature financière, une circulaire ministérielle ${ }^{3}$ 'empresse d'établir une distinction entre les initiatives de formation continue promues au niveau national et provincial d'une part et, d'autre part, les cours confiés aux IRRSAE ${ }^{4}$, à la $\mathrm{BDP}^{5}$ et au $\mathrm{CEDE}^{6}$. Les premières se voient attribuer la part du lion alors que les seconds, instituts régionaux créés pourtant tout spécialement pour la formation continue du personnel enseignant, se partagent les miettes. Peut-être est-ce parce que ces derniers bénéficient d'une certaine autonomie et qu'ils échappent au contrôle serré qu'une partie importante de l'appareil central souhaite et revendique.

Il est regrettable de devoir traiter de la formation continue avec autant d'amertume car c'est un sujet sur lequel reposent encore les espoirs et les attentes d'un grand nombre d'enseignants.

Dans un pays où, à l'exception de quelques rares et heureuses expériences, les enseignants ne reçoivent pas de formation initiale il parait vraiment paradoxal de confier leur formation continue aux seules initiatives génériques des institutions scolaires.

18 En attendant que l'université puisse proposer des cours spécifiques de sciences de l'éducation et organise des départements répondant aux exigences de la récente loi sur les titres universitaires - ce qui semble être possible à compter de l'année académique 1998/1999 - il aurait été préférable, plutôt que de disperser les ressources, de renforcer le rôle des IRRSAE, CEDE et BDP et de remédier à leur fragilité institutionnelle par le biais d'interventions législatives souhaitées de longue date. C'est malheureusement la première option qui a été retenue alors que, de fait, la dispersion des ressources ne peut satisfaire qu'un nombre très limité de structures, les plus fertiles en matière de projets et les plus riches sur les plans logistique et gestionnaire.

19 La dispersion des ressources humaines et financières, d'ores et déjà préoccupante, ne peut que s'aggraver au point d'annuler les profits d'ordre démographique enregistrés ces dernières années.

\section{Tendances de l'enseignement en Europe}

Dans le tableau général des Perspectives de l'enseignement en Europe, élaboré par le Conseil de l'Europe à Strasbourg le 30 août $1989^{7}$ et demeuré actuel, les ministres de l'Éducation ont formulé les recommandations suivantes:

- la formation initiale et la formation continue devraient être considérées [...] comme un ensemble intégré, une éducation permanente ; 
- la formation continue doit répondre de façon équilibrée aux besoins des enseignants et des étudiants mais aussi aux priorités définies par les autorités en charge de l'éducation [...] ;

- au niveau national, régional et local, tout comme dans les écoles, il convient de mettre en place des systèmes souples permettant aux enseignants de suivre une formation continue en dehors de leurs heures de cours ;

- l'organisation de la formation continue devrait, dans la mesure du possible, faire l'objet d'une concertation entre les institutions scolaires, les autorités déléguées et les organismes chargés de répondre aux besoins de formation [... ] ;

- la formation continue doit être suivie et évaluée de façon systématique [...].

Il ressort de ces quelques informations que l'Italie, au-delà des affirmations de principe précédemment citées, n'a encore pleinement retenu ni la valeur de la concertation, ni l'importance de l'évaluation des résultats de la formation; ces deux aspects demeurent irréalisables dans un plan qui s'obstine à ne proposer que des initiatives occasionnelles, des activités dispersées et irrégulières.

L'efficacité de la formation dépend, en somme, d'un plan sérieusement programmé, soutenu par des objectifs précis qui doivent être poursuivis selon des critères de priorités définis et partagés.

\section{NOTES}

1. Conférence permanente des ministres européens de l'Éducation, $\mathrm{XV}^{\mathrm{e}}$ session, Les nouveaux défis pour les enseignants et leur formation, rapports nationaux sur la formation des enseignants, Strasbourg, Conseil de l'Europe, 1987

2. Loi du 11 août 1991, $\mathrm{n}^{\circ} 262$, Dispositions concernant les autorisations syndicales rétribuées et le personnel du secteur scolaire.

3. Circulaire $\mathrm{n}^{\circ} 867 \mathrm{du} 22$ novembre 1991, chapitre A, Application de l'article 2 de la loi 11.8.91, $n^{\circ} 262$, Plan national de formation continue, collaboration des institutions scolaires dotées on non de la personnalité juridique des IRRSAE, BDP et CEDE, instructions administratives et comptables.

4. Istituto Regionale di Ricerca, Sperimentazione Aggiornamento Educativi.

5. Biblioteca di documentazione pedagogica.

6. Centra europeo dell' educazione.

7. Conseil de la coopération cultuelle, Tendances de l'enseignement européen - Tableau général, Conseil de l'Europe, Strasbourg, 1989. 


\section{RÉSUMÉS}

Si de nombreux textes juridiques traitent de la formation initiale et continue des enseignants, en Italie, leur existence ne correspond pas toujours à des réalités positives. Problèmes financiers et problèmes humains aggravent parfois des situations déjà difficiles dues à une politique de dispersion des moyens.

If many legal texts deal with initial teacher training and in-service training in Italy, they do not always correspond to positive realities. Financial and human problems often aggravate situations which are already difficult due to a policy of dispersion of means.

Si bien numerosos textos juridicos tratan de la formación inicial y permanente de los docentes, en Italia, su existencia no corresponde siempre con realidades positivas. Problemas financieros y problemas humanos empeoran a veces situaciones ya dificiles debido a una politica de dispersión de los medios.

\section{INDEX}

Index géographique : Italie

Mots-clés : formation continue, formation des enseignants, formation initiale

\section{AUTEURS}

\section{GIANNI COLOMBO}

Consigliere al Parlemento Europeo per le politiche dell'istruzione e délia formazione 